\title{
On the Belt and Moebius Isomers of the Coronene Molecule
}

\author{
Jan Cz. Dobrowolski ${ }^{\dagger}$ \\ Drug Institute, 30-34, Chełmska Street, 00-725 Warsaw, Poland, and Industrial Chemistry Research Institute, \\ 8, Rydygiera Street, 01-793 Warsaw, Poland
}

Received August 21, 2001

\begin{abstract}
The structure and stability of ca. 100 nonplanar constitutional isomers of the coronene molecule of general formula $\mathrm{C}_{24} \mathrm{H}_{12}$ have been estimated by semiempirical and by ab initio calculations. The isomers studied are unbranched cyclic catacondensed structures and fall into two classes, viz., the belt (B) and the Moebius (M) type. The calculations have shown some of the M-isomers to be more stable than the corresponding B-isomers and thus to form a platform for the future synthesis of or search for such isomers in Nature.
\end{abstract}

\section{INTRODUCTION}

Polycyclic aromatic hydrocarbons (PAHs) are formed during pyrolysis or incomplete combustion of various kinds of organic materials. High-temperature carbonization coal tar is believed to contain some 10000 compounds including the majority of PAHs. ${ }^{1}$ The amount of coronene in coal tar is small, but a mineral called pendletonite (found in California) is $99 \%$ pure coronene. ${ }^{2}$ The discovery of fullerenes ${ }^{3}$ was a milestone not only in the understanding of possible new allotropic forms of polycarbon systems ${ }^{4,5}$ but also a turning-point in the perception of nonplanar PAHs: the nonplanar PAHs comprise evident building motives of nanotubes. ${ }^{6}$ The planar $C_{6 h}$ symmetry coronene molecule can be seen as the limit case of the belt $\mathrm{C}_{24} \mathrm{H}_{12}$ molecule. The other, still unsynthesized and unknown, limit case would be determined by the nonplanar molecule of the $C_{6 h}$ symmetry with six condensed aromatic rings. There has been, however, a considerable progress toward the synthesis of a larger system of $C_{12 h}$ symmetry, viz., the [12]beltene with 12 condensed aromatic rings, involving synthesis of its dodecahydro derivative. ${ }^{7-10}$ To our knowledge, none of the considered Moebius and belt molecules (except for coronene) is so far known.

\section{COMPUTATIONS}

Preliminary molecular modeling, i.e., structure building and AM1 optimization, was performed by using the Spartan 5 package of programs with a graphical interface. ${ }^{17}$ The ab initio calculations were carried out with the Gaussian $98^{18}$ system of programs. Both programs were executed on SGI computers. Full optimization was done at the HF/6-31G** level. For the lowest energy structures, theoretical ${ }^{13} \mathrm{C}$ NMR chemical shifts were calculated using the CHF-GIAO approach ${ }^{19}$ based on the HF/DZP-HB single-point calculations for the HF/6-31G** optimized structures of the molecules. The DZP-HB denotes the double- $\zeta$ DZP-CGTO basis of Hansen and Bouman ${ }^{20}$ composed of (31/7) AO contracted to [2s1p] for hydrogen and of (721/221/1) AO contracted to [3s3p1d] for carbon atom. The double- $\zeta$ basis set ${ }^{21}$ used here

\footnotetext{
$\dagger$ Corresponding author phone: +(48 22) 85152 30; fax: +(48 22) 841 06 52; e-mail: janek@il.waw.pl.
}

has already been shown to be efficient for chemical-shift calculations. ${ }^{22-26}$ Chemical shifts $\left(\delta_{\mathrm{i}}\right)$ were calculated by subtracting the appropriate isotropic part of the shielding tensor $\sigma_{\mathrm{i}}$ from that of TMS $\sigma_{\mathrm{TMS}}: \delta_{\mathrm{i}}=\sigma_{\mathrm{TMS}}-\sigma_{\mathrm{i}}$. The $\sigma_{\mathrm{TMS}}$ values for ${ }^{13} \mathrm{C}$ and ${ }^{1} \mathrm{H}$ were calculated also at the $\mathrm{HF} / 6$ -

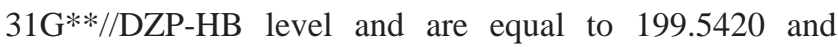
$32.2501 \mathrm{ppm}$, respectively.

Sometimes in course of the AM1 optimization several conformers of one isomers were obtained. In these cases the minimum energy structure was taken for further ab initio calculations. Moreover, sometimes the ab initio symmetry was lower than MM or AM1 symmetry which suggests that a potential barrier between more than one lower symmetry minimum exists. This problem was not further explored.

\section{RESULTS AND DISCUSSION}

3.1. Structures. 3.1.1. Number and Code of Coronene Isomers. The unbranched cyclic catacondensed constitutional coronene isomers of the belt type (B) can be derived from the unbranched catacondensed hexacene isomers, of the general formula $\mathrm{C}_{26} \mathrm{H}_{16}$, by assuming the pairs of $\mathrm{HC}-\mathrm{CH}$ moieties, from the first and sixth six-membered rings, to be deprotonated and fused together into a pair of carbon atoms $>\mathrm{C}-\mathrm{C}<$ binding these rings. In this way a compound of the general formula $\mathrm{C}_{24} \mathrm{H}_{12}$ can be formed (Figure 1).

An extensive way to find all constitutional $\mathrm{B}$-isomers of coronene is the following: For each out of the 25 unbranched catacondensed hexacene isomers nine different glueing types can be formed (Figure 1). There are three different $\mathrm{CH}-$ $\mathrm{CH}$ bonds in the first and in the last cycle of the catacondensed structure. By glueing them together, nine structures are formed, usually not all of them different. Successive consideration of all of them, and elimination of those that have been previously considered, allows one to discover all the 52 different coronene belt isomers.

Another way to reckon the number of B coronene isomers can be based on the code of the belt structure. Let us note that each $\mathrm{B}$-isomer is formed by six six-membered rings. Each B-isomer has two boundaries formed by two sequences of six of the following moieties: $(\mathrm{CH})_{2},(\mathrm{CH})_{1}$, or $(\mathrm{CH})_{0}$ (single bond). Each of the $(\mathrm{CH})_{k}$ moieties in the B-isomer can be represented by the number $k=2,1$, or 0 , respectively, 


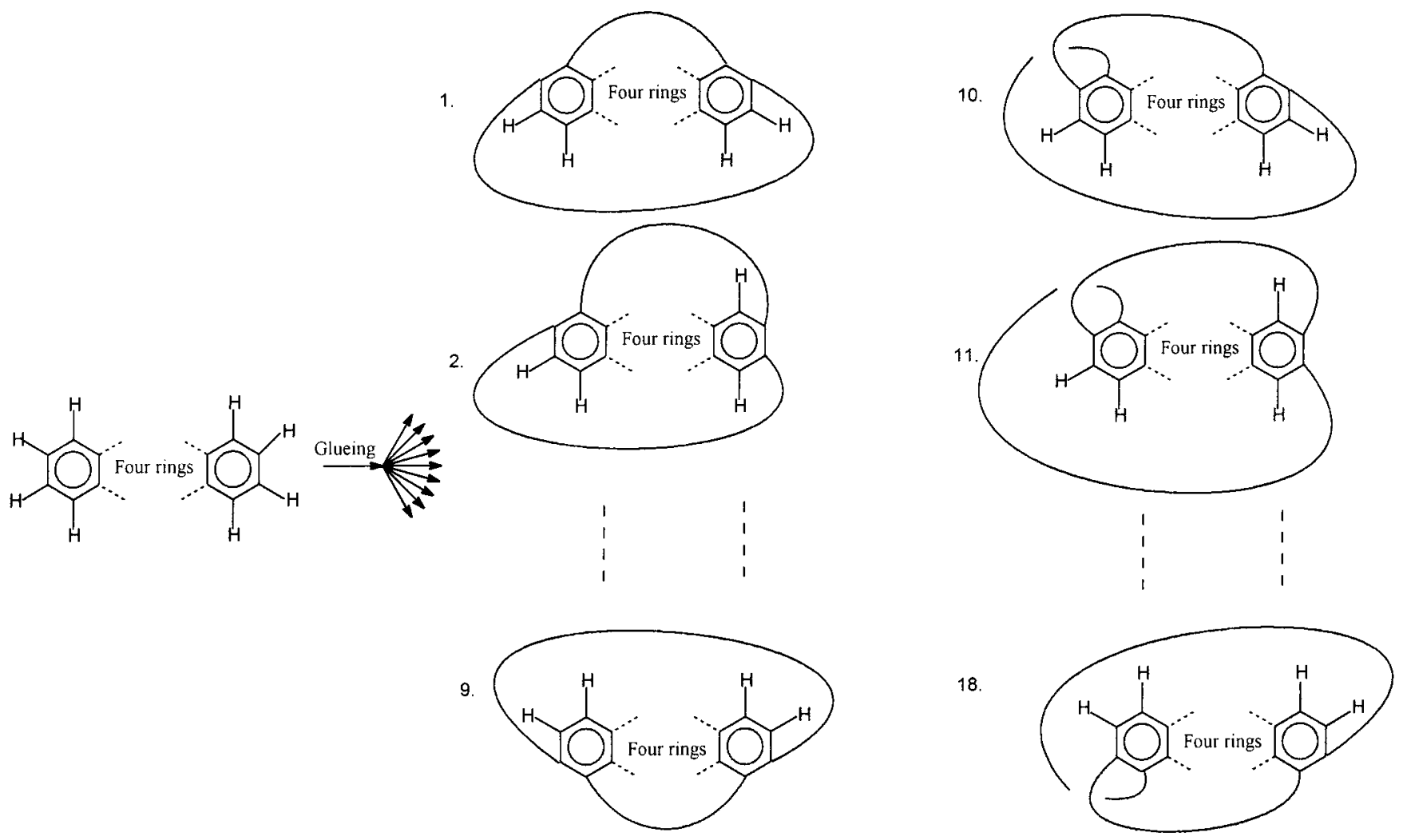

Figure 1. The belt and Moebius coronene isomers can be developed from catacondensed hexacene isomers by glueing two pairs of $\mathrm{CH}-$ $\mathrm{CH}$ bonded atoms from the first and the sixth ring into a single pair of $\mathrm{C}-\mathrm{C}$ bonded atoms binding the resultant system into a belt or a (half-twisted) Moebius strip.

and for each B-isomer two six-tuples can be written as $\left(\mathrm{a}_{1}\right.$, $\left.\mathrm{a}_{2}, \mathrm{a}_{3}, \ldots, \mathrm{a}_{6}\right)$ and $\left(\overline{\mathrm{a}}_{1}, \overline{\mathrm{a}}_{2} \overline{\mathrm{a}}_{3}, \ldots, \overline{\mathrm{a}}_{6}\right)$, where $\mathrm{a}_{\mathrm{i}}=k$ when the $i$ th ring yields the $(\mathrm{CH})_{k}$ moiety to the boundary, $k=0,1,2 . \bar{a}_{\mathrm{i}}$ $=2,1$, or 0 when the entry in the first tuple is $a_{i}=0,1$, or 2 , respectively.

Let us now introduce an equivalence relation between the tuples: Two six-tuples are said to be equivalent if and only if they describe the same B-isomer. Indeed, this is the equivalence relation because it is reflexive (each six-tuple is in relation with itself, because it describes the same structure), symmetric (if a six-tuple $\boldsymbol{A}$ is in relation with a six-tuple $\boldsymbol{B}$, because they describe the same structure, then $\boldsymbol{B}$ is in relation with $\boldsymbol{A}$ ), and transitive (if a six-tuple $\boldsymbol{A}$ is in relation with a six-tuple $\boldsymbol{B}$, and $\boldsymbol{B}$ is in relation with a sixtuple $\boldsymbol{C}$, then $\boldsymbol{A}$ is in relation with $\boldsymbol{C}$ ). First, note that both six-tuples that describe two boundaries of the same structure are usually different and yet they describe the same structure and therefore they are equivalent. Next, the first ring is taken as an arbitrary ring, and therefore each cyclic permutation of the six-tuple, clockwise and counterclockwise, describes the same boundary and the appropriate six-tuples are equivalent. Finally, the number of abstract classes of the equivalence relation among the six-tuples is deduced to be 52. The codes of the belt structures are the first six digits of the 12-tuples given in Table 1. The belt structure can be chiral: e.g., the B-structure (111102) and its mirror image is not superimposable; however, this fact does not produce any splitting in the B-isomer energies. Chirality can be detected for all the B-structures that belong to the $S_{n}$ group of symmetry, in other words for $24 \mathrm{~B}$-isomers that do not belong to the $D_{6 h}, C_{3 V}, C_{2 V}, C_{2 h}$, and $C_{\sigma}$ group of symmetry.
Thus including chirality, the number of different B-isomers is $2 \cdot 24+28=76$.

The Moebius constitutional coronene isomers are all chiral: they can be half-twisted "to the left" or "to the right", and none of them has symmetry higher than $C_{2}$. Here, we ignore the chirality of the $\mathrm{M}$-isomer structures and represent the pair of the optical isomers by one representive, only. To count the number of different $\mathrm{M}$ coronene isomers let us first see that a half-twisted Moebius coronene isomer has one boundary only. Therefore, the code analogous to that constructed for the B-isomers contains 12 entries. However, the $\mathrm{M}$-isomers code has a specific composition connected with the M-isomer building motif, the six-membered ring, and therefore it can be written in the following form

$$
\left(\mathrm{a}_{1}, \mathrm{a}_{2}, \mathrm{a}_{3}, \ldots, \mathrm{a}_{6}, \overline{\mathrm{a}}_{1}, \overline{\mathrm{a}}_{2}, \overline{\mathrm{a}}_{3}, \ldots, \overline{\mathrm{a}}_{6}\right)
$$

where the symbols have the same meaning as those for B-isomer codes.

An analogous equivalence relation between 12-tuples can be introduced for the $\mathrm{M}$ coronene isomers: Two 12-tuples are said to be equivalent if and only if they describe the same M-isomer. As before, this is equivalence relation: reflexive, symmetric, and transitive. It can undergo both clockwise and counterclockwise cyclic permutation of the whole 12-tuple; for example, the following 12-tuples are equivalent: $\left(\mathrm{a}_{1}, \mathrm{a}_{2}, \mathrm{a}_{3}, \ldots, \mathrm{a}_{6}, \overline{\mathrm{a}}_{1}, \overline{\mathrm{a}}_{2}, \overline{\mathrm{a}}_{3}, \ldots, \overline{\mathrm{a}}_{6}\right) /\left(\overline{\mathrm{a}}_{5}, \overline{\mathrm{a}}_{6}, \mathrm{a}_{1}, \mathrm{a}_{2}\right.$, $\left.\mathrm{a}_{3}, \ldots, \mathrm{a}_{6}, \overline{\mathrm{a}}_{1}, \overline{\mathrm{a}}_{2}, \overline{\mathrm{a}}_{3}, \ldots, \overline{\mathrm{a}}_{4}\right)$.

The number of M-isomers is the number of the abstract classes of the equivalence relation between the 12-tuples, and it is found to be equal to 45 . To find this number, let us 
Table 1. AM1 Heats of Formation Differences $\left(\Delta \mathrm{H}_{\mathrm{f}}\right)$ and SCF Total Energy Differences $(\Delta \mathrm{E})$ for Belt $(\mathrm{B})$ and Moebius $(\mathrm{M})$ Coronene Isomers $^{a}$

\begin{tabular}{|c|c|c|c|c|c|c|c|c|c|c|c|}
\hline \multirow[t]{2}{*}{$\#$} & \multirow{2}{*}{ Structure scheme } & \multirow{2}{*}{ Code } & \multirow{2}{*}{ Proposed Name } & \multicolumn{4}{|c|}{$\begin{array}{c}\text { AM1 } \\
\mathrm{kcal} / \mathrm{mol}\end{array}$} & \multicolumn{2}{|c|}{$\begin{array}{c}\mathrm{HF} / \mathrm{STO} \text {-3G } \\
\mathrm{kcal} / \mathrm{mol}\end{array}$} & \multicolumn{2}{|c|}{$\begin{array}{c}\mathrm{HF} / 6-31 \mathrm{G}^{* *} \\
\mathrm{kcal} / \mathrm{mol}\end{array}$} \\
\hline & & & & B & & $\mathbf{M}$ & & B & $\mathbf{M}$ & B & $\mathbf{M}$ \\
\hline 1 & & 11111111111 & Belt-[11]-hexacene & 297.76 & $\mathrm{D}_{6 \mathrm{~h}}$ & $n c$ & & 426.040 & $n c$ & 359.410 & $n c$ \\
\hline 2 & & $111110 \quad 111112$ & Belt-[10]-hexacene & 241.49 & $C_{0}$ & 398.92 & & 342.802 & 531.357 & 308.945 & 473.405 \\
\hline 3 & & $111102 \quad 111120$ & Belt-[02]-hexacene & 250.82 & $\mathrm{C}_{2}$ & $n c$ & $\mathrm{C}_{2}$ & 356.498 & $n c$ & 321.198 & $n c$ \\
\hline 4 & & $111100 \quad 111122$ & Belt-[00]-hexacene & 237.36 & $C_{o}$ & 305.66 & & 335.069 & 422.842 & 300.342 & 376.794 \\
\hline 5 & & 111012111210 & Belt-[12]-benzo[a]pentacene & 236.74 & $\mathrm{C}_{2}$ & 433.63 & & 339.946 & 573.164 & 307.579 & 507.126 \\
\hline 6 & & 111010111212 & Belt-[10]-benzo[a]pentacene & 218.50 & $\mathrm{C}_{0}$ & 339.25 & & 308.076 & 451.674 & 278.072 & 403.436 \\
\hline 7 & & 110112112110 & Belt-[12]-hexaphene & 230.62 & $\mathrm{C}_{2 \mathrm{~h}}$ & 418.94 & $\mathrm{C}_{2}$ & 330.663 & 516.711 & 300.401 & 461.791 \\
\hline & & 110110112112 & Belt-[10]-hexaphene & 215.65 & $\mathrm{C}_{2 \mathrm{v}}$ & & & 305.742 & & 278.199 & \\
\hline 8 & & M-code $\equiv 7$ & Moeb-[12]-hexaphene & & & & & & & & \\
\hline 9 & & 111020111202 & Belt-[20]-benzo[a|pentacene & 231.21 & $\mathrm{C}_{\sigma}$ & 452.17 & & 327.295 & 597.702 & 295.420 & $n c$ \\
\hline 10 & & 111002111220 & Belt-[02]-benzo[a]pentacene & 242.42 & & 330.74 & & 343.310 & 490.439 & 308.447 & 439.398 \\
\hline 11 & & $111000 \quad 111222$ & Belt-[00]-benzo[a]pentacene & 224.21 & $\mathrm{C}_{2}$ & 229.72 & & 313.907 & 329.601 & 279.368 & 292.209 \\
\hline 12 & & $110120 \quad 112102$ & Belt-[20]-hexaphene & 225.44 & & 448.40 & & 318.361 & 593.830 & 287.045 & 497.200 \\
\hline 13 & & 110102112120 & Belt-[02]-hexaphene & 227.67 & & 364.72 & & 321.050 & 484.747 & 289.111 & 433.821 \\
\hline 14 & & 110012112210 & Belt-[12]-naphtho[1,2-a]naphthacene & 231.38 & & 314.00 & & 329.369 & 428.346 & 296.371 & 385.219 \\
\hline 15 & & $110010 \quad 112212$ & Belt-[10]-naphtho[1,2-a]naphthacene & 202.58 & & 266.19 & & 288.733 & 371.637 & 260.348 & 332.701 \\
\hline 16 & & 101012121210 & Belt-[12]-benzo[a]pentaphene & 220.61 & $C_{\sigma}$ & 322.76 & & 315.516 & 441.979 & 283.818 & 396.530 \\
\hline $60^{\circ}$ & & 101210121012 & Moeb-[10]-benzo[c]pentaphene & & & 435.73 & & & 552.849 & & 507.449 \\
\hline & & $101010 \quad 121212$ & Belt-[10]-benzo[a]pentaphene & 194.21 & $\mathrm{C}_{3 v}$ & & $\mathrm{C}_{2}$ & 280.278 & & 257.015 & \\
\hline 17 & & M-code $\equiv 16$ & Moeb-[12]-benzo[a]pentaphene & & & & & & & & \\
\hline 18 & & $110220 \quad 112002$ & Belt-[20]-naphtho[2,1-a] naphthacene & 246.18 & $\mathrm{C}_{\sigma}$ & 542.95 & & 346.564 & $n c$ & 314.237 & $n c$ \\
\hline
\end{tabular}


Table 1 (Continued)

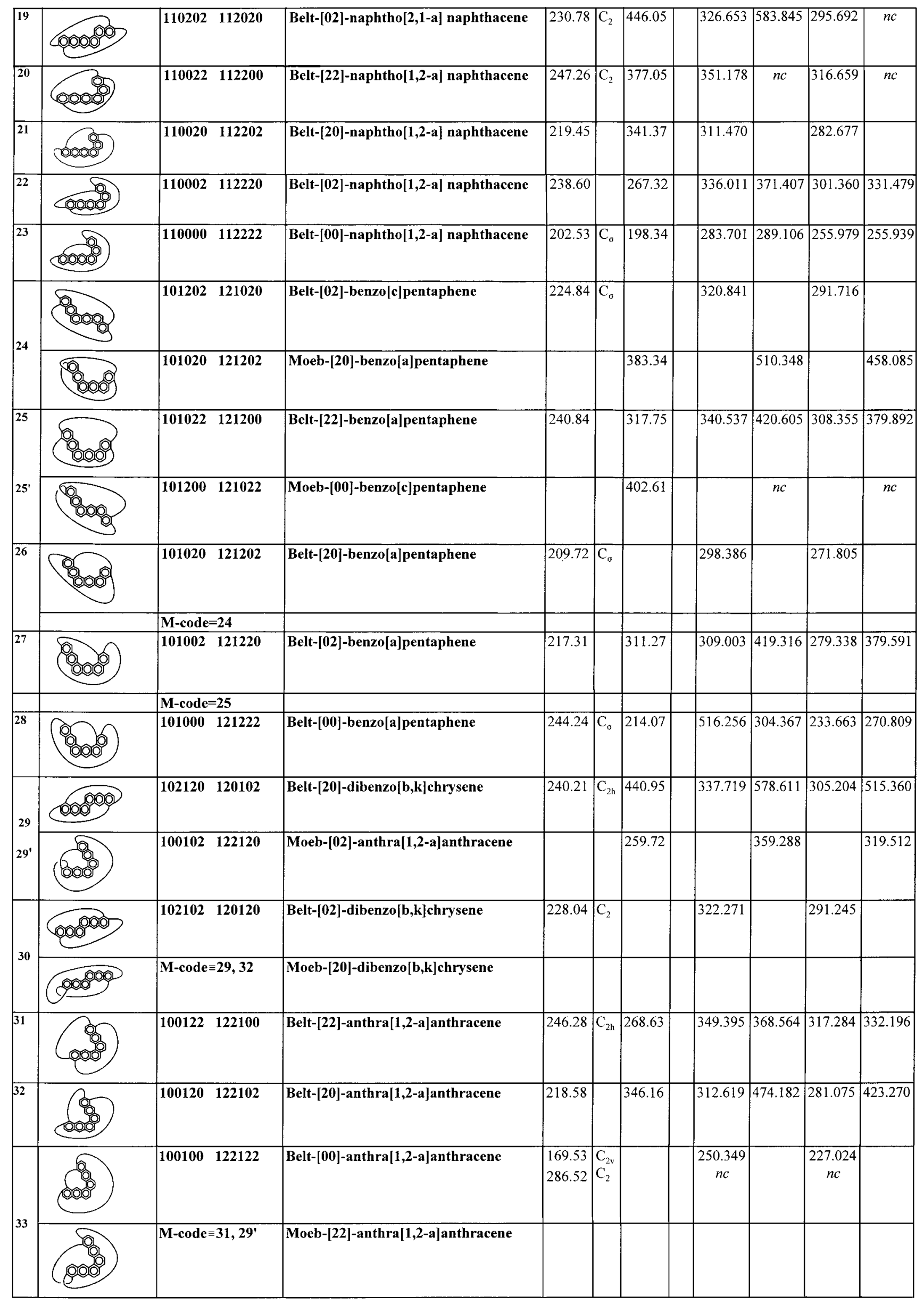


Table 1 (Continued)

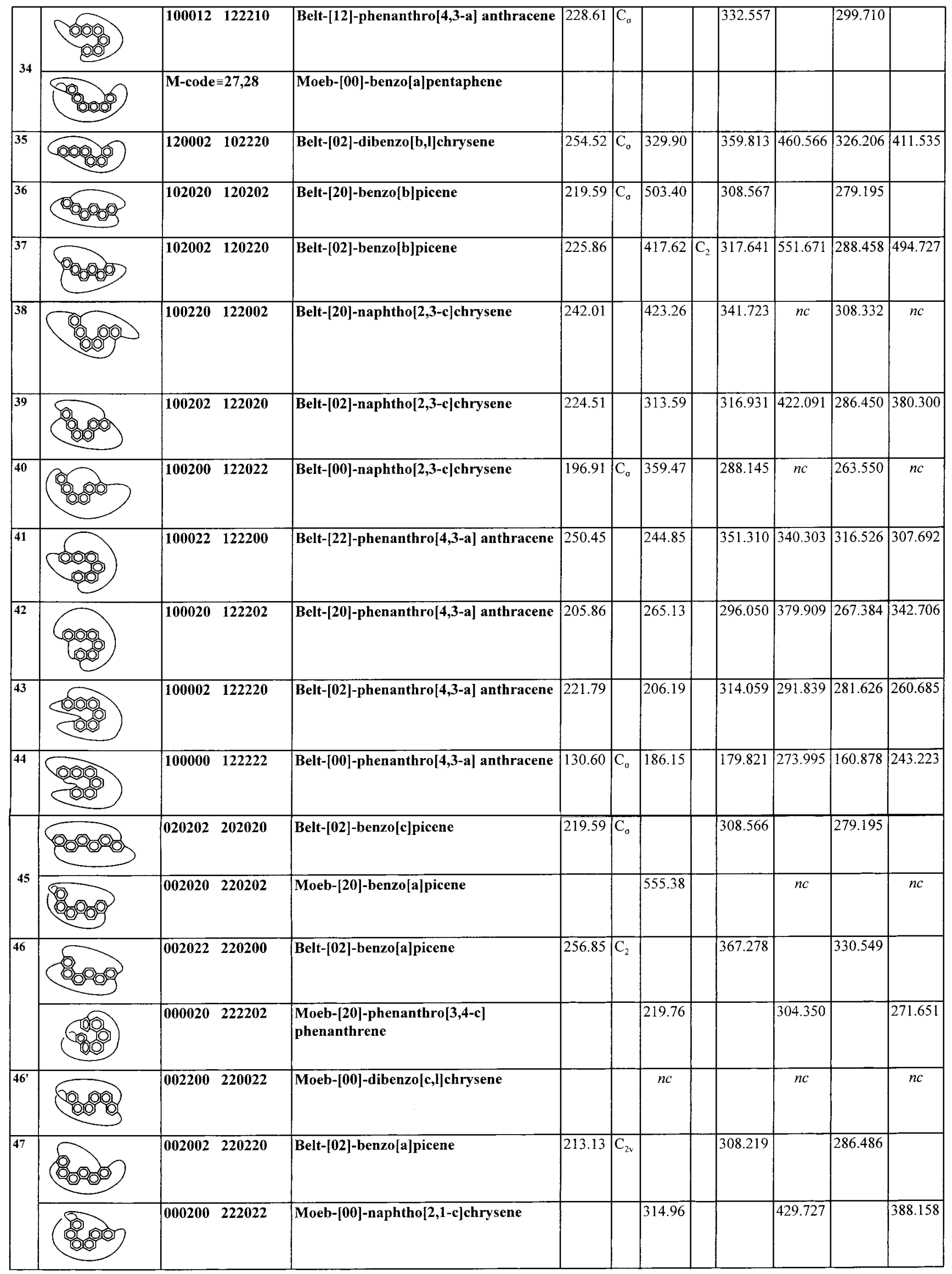


Table 1 (Continued)

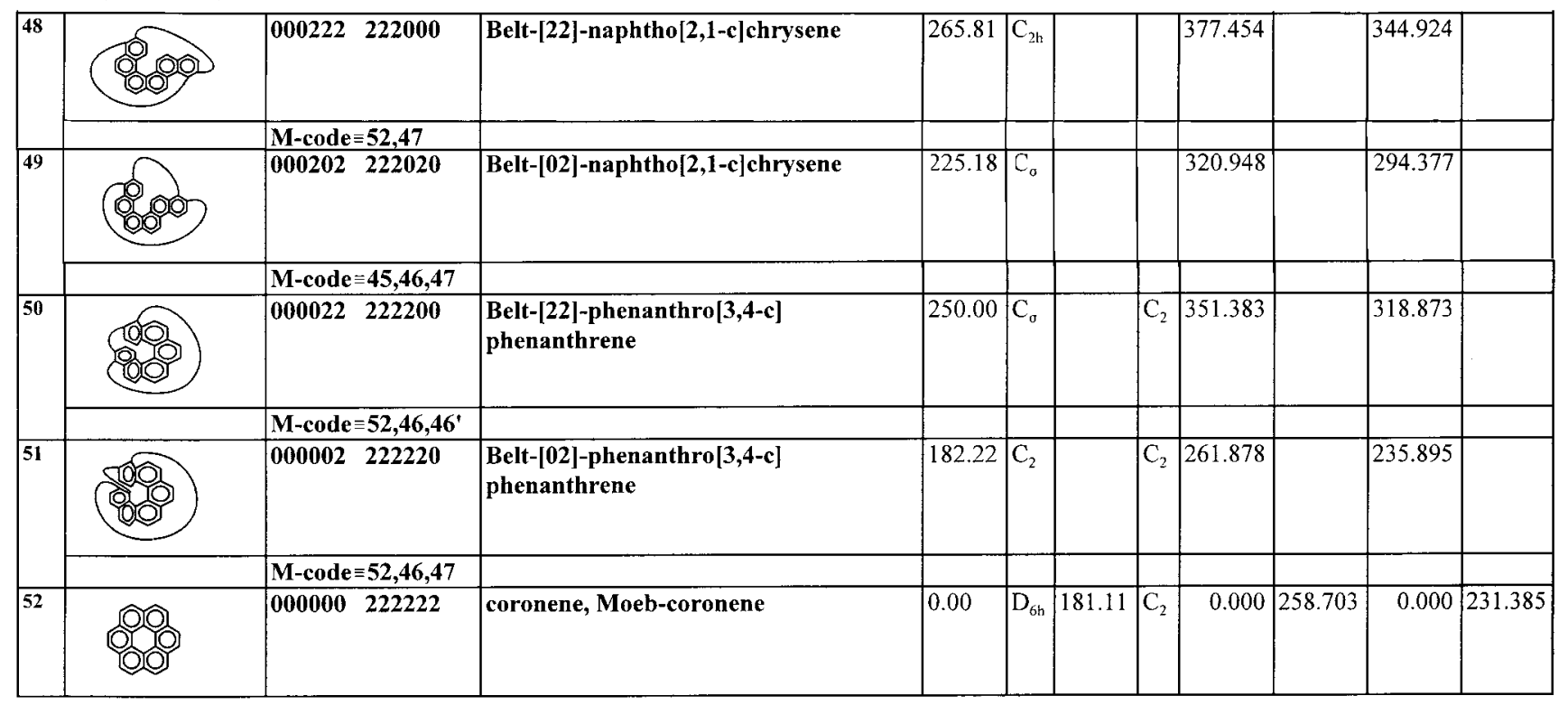

${ }^{a} \Delta \mathrm{E}=\mathrm{E}_{\mathrm{i}} \mathrm{E}$ (coronene), $\mathrm{H}_{\mathrm{f}}$ or $\mathrm{E}$ (coronene) $=96.23 \mathrm{kcal} / \mathrm{mol}$, or -904.822707 and -915.974077 hartree, with the AM1 method or STO-3G and 6-31G** basis set, respectively. 1 hartree $=627.5095 \mathrm{kcal} / \mathrm{mol}, n c$ stands for not converged. The accuracy $0.001 \mathrm{kcal} / \mathrm{mol}$ is modest, and for the total energy can be recalculated to within six decimal digits.

first see that an M-isomer can be developed from a B-isomer by breaking the bonds between two six-membered rings and rebinding them "upside down". Second, there are six ways to break the B-isomer without changing the six-tuple code: one can break and rebind the bond "upside down" between the first and the second ring, second and third, ..., sixth and first. (If the B-isomer is broken with a change in the code, the situation falls into the other, code preserving, cleavage.) Now, the codes (Table 1) fall into separate classes determined by the number of identical digits in the code and subclasses with respect to the neighboring of digits. The equivalence relation can occur solely between the codes from the same class and subclass. This remark allows for the reduction of the number of codes to be considered when searching for equivalent structures. For example, the codes (111100 111122), (111102 111120), (111010 111212), (111012 111210), (110110 112112), and (110112 112110) form a sixelement class. Moreover, a cyclic permutation of digits in the last two 12-tuples shows that they are equivalent even though, for the B-isomers, they describe two different structures. Analogously, the codes containing six digits one form a nine-tuple class. The latter fall into three subclasses: in the first (three-element), three digits one are neighboring, in the second (four-element), two digits one are neighboring, and the third digit one is separated by one or two other digits and, in the third subclass (two-element), digits one are no neighbors whatsoever. The third subclass is interesting: two codes that described B-isomers, (101012 121210) and (101010 121212), are equivalent by 12-tuples cyclic permutation. However, by "breaking and rebinding" the corresponding B-isomers, a new element can be found: (101210 121012). It represents the same B-isomer as does the code (101012 121210): cyclic permutation of the first six digits in the last code and subsequent permutation of the second six digits gives the code identified above, and yet, as 12tuples, they are not equivalent. Thus, the equivalence relation between the 12-tuples belonging to one subclass can occur even if there is no equivalence between the component sixtuples determining the related B-isomer. On the other hand, considering the isomers that can be formed from the B-isomer by "breaking and rebinding", a new 12-tuple can be found despite the nonequivalence between 6-tuples. The latter situation can be demonstrated as follows: Let us consider the code of the B-structure 32: (100120 122102) and let us perform "breaking and rebinding" of the structure. This corresponds to successive permutation of the first and second six-tuples of the B-code:

(100120 122102) structure 32

(001201 221021) structure 32 (optical isomer)

(012010 210212) structure 30

(120100 102122) structure 29'

(201001 021221) structure 29'

(010012 212210) structure 33 (optical isomer)

Finally, considering all the possibilities, one can conclude that the number of the abstract classes of the equivalence relation between the 12-tuples is 45 (if chirality is ignored but including chirality the number is 90). The codes of the M-structures are given in Table 1.

According to a suggestion of the Reviewer of this article, the notation of the chiral M-isomers can be completed by adding to the code the subscript $x$ which is + or - when the M-isomer is twisted to the right or to the left, respectively. Moreover, when a multiple twist is considered, always to the same side, then multiple subscripts can be added, for example $(111102111120)_{+++}$or $(111102111120)_{-}-{ }_{-}$.

The analogous notation of the chiral B-isomers also may be completed by adding the subscript $x$ to the code which is + or - when the B-isomer is chiral and which is 0 when the structure is not chiral, for example (111102)_ or 
Table 2. Order and Codes of the Unbranched Catacondensed Hexacene Isomers
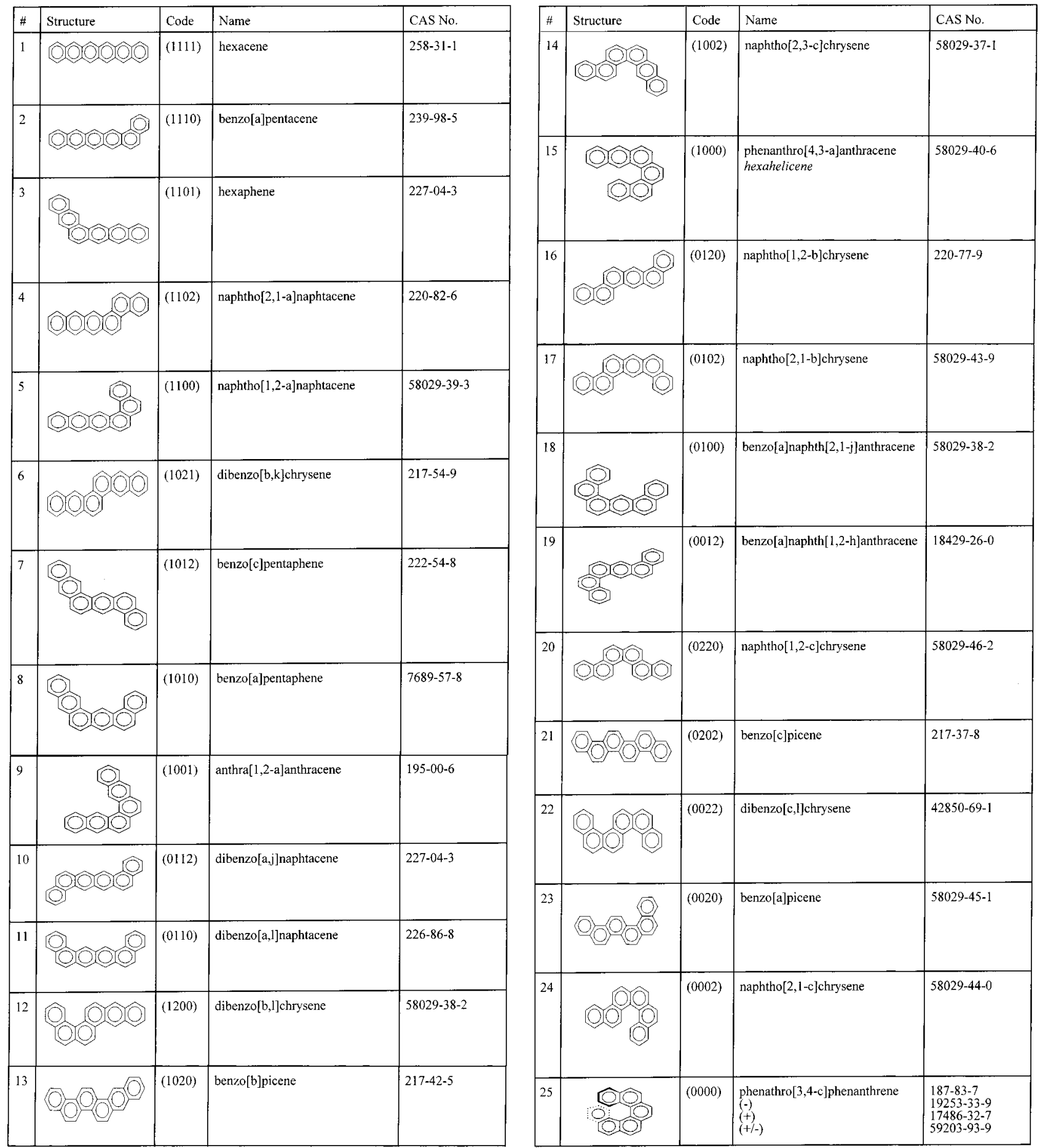

$(111102)_{+}$and $(111110)_{0}$. However, the desired notation of the chiral isomers should refer not only to the twist but also to the Cahn, Ingold, Prelog rules, generalized for the B- and M-isomers studied here. Despite that, introduction of such generalized rules requires a deeper discussion of the relations between twist and $\mathrm{R}$ or $\mathrm{S}$ configuration in $\mathrm{M}$-isomers and proper distinguishing of $\mathrm{R}$ and $\mathrm{S}$ configuration in B-isomers, the tasks which are much beyond of the frames of this paper.

3.1.2. Chemical Names Proposed. The B-isomers of coronene molecule, $\mathrm{C}_{24} \mathrm{H}_{12}$, can be named after the unbranched catacondensed isomers of hexacene molecule of the general formula $\mathrm{C}_{26} \mathrm{H}_{16}$, where two $\mathrm{HC}-\mathrm{CH}$ moieties from the first and the sixth six-membered rings were deprotonated and glued together (cf. last section). The glued carbon atoms are in this paper symbolically bound by two arcs (Figure 1, Table 1). This graphical representation is not unique, and different representations correspond to different cyclic permutations of six-tuple coding the same structure (Figure 2). For each catacondensed hexacene isomer the glued bonds can form nine pairs and the same B-isomer can be named after many different "parent" hexacene isomer structures. Therefore, to avoid ambiguous names, it is necessary to order the catacondensed hexacene isomers. Once the ordering is introduced, isomers can be discerned, which 


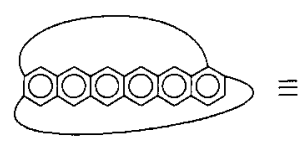

(111110 111112)

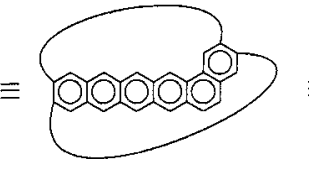

(111101 111121)

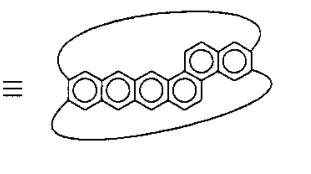

(111011 111211)

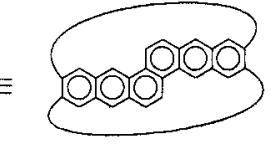

$(110111$ 112111)

Figure 2. The four schemes of belt structures represent the same belt isomer.
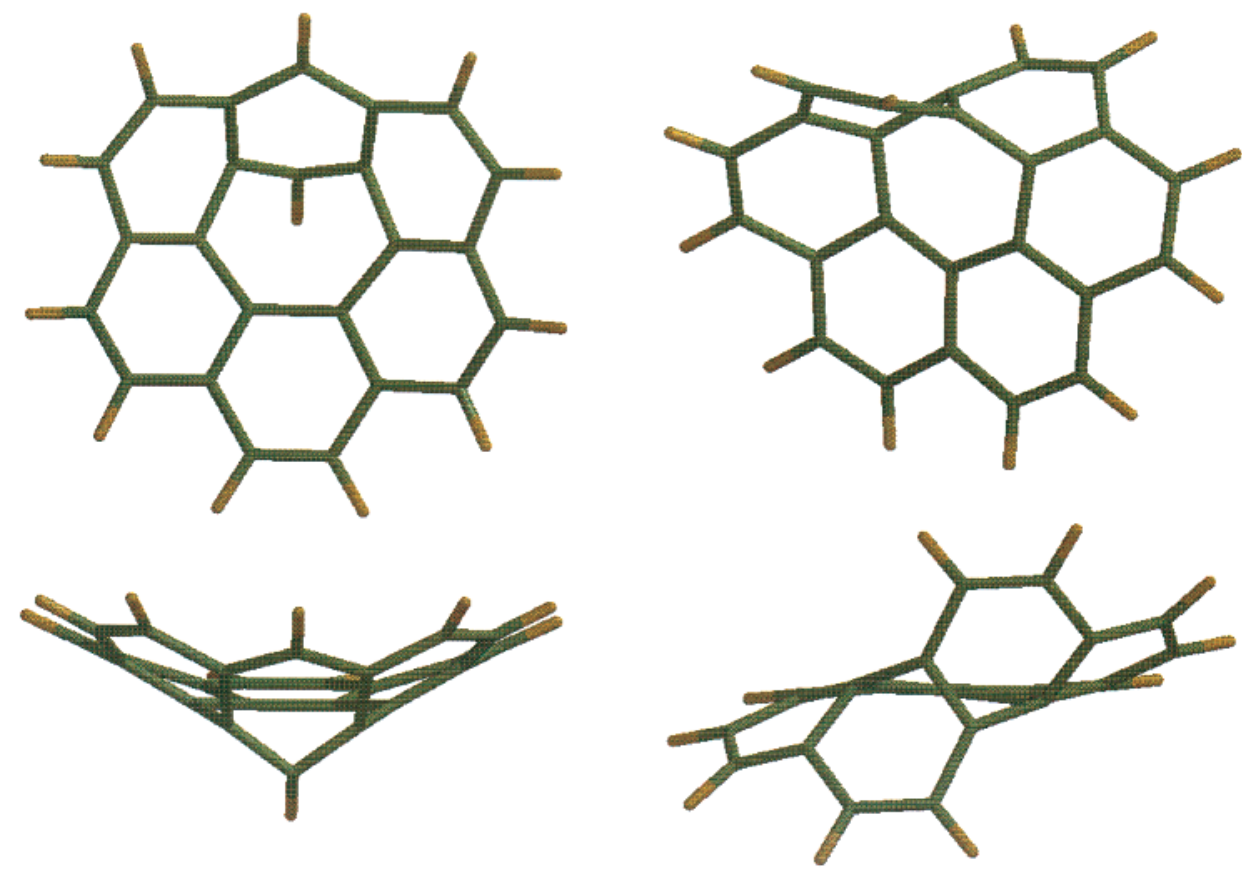

Figure 3. The HF/6-31G** structures of the second most stable belt structure (44) Belt-[00]-phenanthro [4,3-a] anthracene (100000 122222) (A) and the most stable Moebius structure (52) Moeb-coronene (000000 222222) (B).

is smaller (simpler) and which is larger (more complex). The name should be derived from as simple a hexacene isomer as possible. The systematic names of all catacondensed hexacene isomers are not widely known, and therefore, they are included in Table 2. In the Appendix section one can find a definition of the ordering relation among the codes of hexacene isomers.

Now, the six- and the 12-tuples can be ordered by applying the rules for ordering hexacene isomers (Appendix). Assume that the first four digits in a six-tuple originate from an appropriate hexacene isomer and the fifth and the sixth describe the bounding between the sixth rings, respectively. Thus, the three digits at the last two entries in the code produce nine possible situations.

Let us now agree that the B- or M-isomer name will be written in the following form: Belt- $[p q]$-(hexacene isomer name) or Moeb-[pq]-(hexacene isomer name), respectively, where $p$ and $q$ stand for 1,0 , and 2 .

According to the above assumptions and agreements, the B-isomers with the codes (111111), (111110), (111100), and (111102) are named Belt-[11]-, Belt-[10]-, Belt-[00]-, and Belt-[02]- hexacene, respectively. Analogously, the Bisomers with codes (120002) and (102002) are named Belt$[02]$-dibenzo[b,1]chrysene and Belt-[02]-benzo[b]picene, respectively. The names of $\mathrm{M}$-isomers are formed analogously (Table 1); however, according to the given rules some 12tuples have to be written in the form different than the parent $\mathrm{B}$-isomer and, in consequence, some $\mathrm{M}$-isomers have the name derived from a different hexacene isomer (see e.g., structures 45, 46, and 47).

Finally, let us mention that we considered an alternative proposal to symbolize the way of glueing the hexacene isomer by using the letter labels of the hexacene isomer bonds. In such a notation the codes (111111), (111110), (111100), and (111102) would be named Belt- $[b=o]-$, Belt$[b=p]-$, Belt- $[a=p]$-, and Belt- $[a=n]$ - hexacene, respectively. However, the letters changes from one structure to another much more than does the number of $\mathrm{CH}$ bond in the structure. This is the way to propose to derive the name with the last two digits of the code rather than the letter labels of the hexacene isomer bonds.

3.2. Energetics. 3.2.1. Energy Difference: Belt and Moebius versus Coronene Structure. So far, the coronene molecule (52) is the only molecule known to exist from among all the structures described. Therefore, the belt and the Moebius isomer energy is referred to the energy of the coronene molecule (Table 1). The energy difference is quite large: its minimum value (at the $\mathrm{HF} / 6-31 \mathrm{G}^{* *}$ level, Table 1) is for the belt isomers equal to ca. $160 \mathrm{kcal} / \mathrm{mol}$ (44) and, for Moebius isomers, equal to ca. $231 \mathrm{kcal} / \mathrm{mol}$ (52). There are only a few other belt isomers $(\mathbf{1 5}, \mathbf{1 7}, \mathbf{2 3}, \mathbf{2 6}, \mathbf{2 8}, \mathbf{3 3}, \mathbf{4 0}$, 42, 51) and still fewer Moebius structures (23, 28, 43, 44, 46) whose energy difference does not exceed $275 \mathrm{kcal} / \mathrm{mol}$. However, it should be borne in mind that there are various reaction paths and that high energy alone cannot exclude the possibility of the existence of a high-energy structure. 
Notice that the AM1 method suggests the B- and M-isomers stabilities higher than does the HF/6-31G** method, and the latter predicts higher stabilities are than those from the HF/ STO-3G method.

The above-mentioned six low-energy $\mathrm{M}$-isomers are more stable than the $42 \mathrm{~B}$-isomers of stabilization energies higher than $275 \mathrm{kcal} / \mathrm{mol}$ and, therefore, should be easier to obtain than those B-isomers. Moreover, there are four M-isomers $(\mathbf{2 3}, \mathbf{4 1}, \mathbf{4 3}, \mathbf{4 6})$ that have smaller energies than the B-isomers with the analogous codes. In these particular cases, the $\mathrm{M}$-isomers should be formed more easily than the B-isomers.

Finally, all the $52 \mathrm{~B}$-isomers converged to their minimum energy structures, while several M-isomers converged to the structures with formation of three-, four-, or five-membered rings with additional $\mathrm{C}-\mathrm{C}$ bonds or spiro carbon atoms, and therefore their energies are not listed in Table 1.

3.3. Qualitative NMR Spectra. 3.3.1. Symmetry. Many of the structures studied can have symmetry elements. In fact, during the course of optimization, those symmetry elements are often lost, and the optimized form exhibits a lower symmetry than the preoptimized MM-structure. Nevertheless, the point group of symmetry to which a structure may belong is listed in Table 1 in the column with AM1 heat of formation.

3.3.2. Qualitative NMR Spectra. Two $D_{6 h}$ symmetry structures, coronene (52) and Belt-[11]-hexacene (1) molecules, exhibit the simplest theoretical ${ }^{1} \mathrm{H}$ NMR and ${ }^{13} \mathrm{C}$ NMR spectra. The coronene ${ }^{13} \mathrm{C}$ NMR spectrum ${ }^{27}$ consists of three signals corresponding to the central ring six quaternary carbon atoms at $122.67 \mathrm{ppm}$, the six quaternary carbon atoms at $128.81 \mathrm{ppm}$, and the $12 \mathrm{C}(\mathrm{H})$ atoms at the $126.20 \mathrm{ppm}$ (measurements in $\mathrm{CDCl}_{3}$ ). The calculations predict the signals to be placed at $132.70,137.56$, and 134.70 ppm, respectively. Thus the ${ }^{13} \mathrm{C}$ NMR signals are calculated to be placed at $10 \mathrm{ppm}$ lower field than experimental data. Such a discrepancy is acceptable if the qualitative conclusions are to be drawn only. The coronene ${ }^{1} \mathrm{H}$ NMR spectrum ${ }^{27}$ consists of one signal placed at $8.92 \mathrm{ppm}\left(\mathrm{CDCl}_{3}\right)$ and it is predicted to be located at $9.34 \mathrm{ppm}$. Note, that the surrounding was not simulated in the calculations, and therefore the $0.4 \mathrm{ppm}$ of difference between theoretical and experimental data is plausible. The predicted ${ }^{13} \mathrm{C}$ NMR spectrum of Belt-[11]-hexacene consists of two signals placed at 170.13 and $154.67 \mathrm{ppm}$, which correspond to quaternary carbon and $\mathrm{C}(\mathrm{H})$ carbon atoms, respectively. The only one ${ }^{1} \mathrm{H}$ NMR signal of the Belt-[11]-hexacene spectrum is placed at 3.41 $\mathrm{ppm}$, in a region rather not typical to unsaturated hydrocarbons, the position which suggests the aromatic structure to be very strained.

The Belt-[00]-phenanthro[4,3-a]anthracene (100000 122222) (44) is the second most stable B-structure which exhibits the $C_{\sigma}$ symmetry. Therefore, the number of NMR signals is reduced twice where atoms are placed outside the symmetry plane. The predicted positions of the ${ }^{13} \mathrm{C}$ NMR signals are the following: six quaternary carbon atoms: $159.56,147.58$, $145.98,143.33,135.00,129.43 \mathrm{ppm}$; five $\mathrm{C}(\mathrm{H})$ atoms placed out of the symmetry plane: $136.96,135.96,134.82,133.37$; $127.36 \mathrm{ppm}$, and two $\mathrm{C}(\mathrm{H})$ atom placed at the symmetry plane: 144.44 and $141.00 \mathrm{ppm}$. The predicted five ${ }^{1} \mathrm{H}$ NMR signals of protons placed out of the symmetry plane are 7.83 , 7.76, 7.75, 7.16, $6.88 \mathrm{ppm}$, and the two ${ }^{1} \mathrm{H}$ NMR signals of protons placed at the symmetry plane are predicted at 10.89 and $8.00 \mathrm{ppm}$.

The Moeb-coronene (000000 222222) (52) is the most stable M-structure and possesses $C_{2}$ symmetry. Therefore, the number of NMR signals is reduced twice where atoms are placed outside the 2 -fold symmetry axis. The predicted positions of the ${ }^{13} \mathrm{C}$ NMR signals are the following: five quaternary carbon atoms placed out of the symmetry axis: $156.91,150.19,144.76,141.80 ; 136.03 \mathrm{ppm}$; and two quaternary carbon atoms placed at the symmetry axis: 141.53 and $137.24 \mathrm{ppm}$, and six $\mathrm{C}(\mathrm{H})$ atoms: 143.67, 137.13, $136.77,136.08,131.59$, and $131.40 \mathrm{ppm}$. The predicted six ${ }^{1} \mathrm{H}$ NMR signals of protons placed out of symmetry axis are the following: $7.94,786,7.47,7.47,7.01$, and $6.75 \mathrm{ppm}$.

\section{CONCLUSIONS}

The planar $C_{6 h}$ symmetry coronene molecule is predicted to produce ca. 100 nonplanar topological isomers of the belt and Moebius type. Those, yet undiscovered, molecules have been enumerated, based on the molecular code, viz., sixtuple for the belt and 12-tuple for the Moebius type of compounds. If chirality of the Moebius isomers is ignored, the number of possible belt and Moebius structures is just the number of the different (suitable) equivalence classes of 6-tuples and 12-tuples, and these numbers are found to be 52 and 45 , respectively. The smaller number of the 12- than that of the 6-tuples originates from the code composition and relation between the digits in the code. Based on the codes and systematic names of the catacondensed hexacene isomers, proposed names for coronene isomers were developed. The ab initio HF/6-31G** calculations showed all the belt coronene isomers to be stable; however, ca. 15 Moebius coronene isomers converged to the structures with altered atom connectivity. For the most stable belt and Moebius structures, the ${ }^{13} \mathrm{C}$ and ${ }^{1} \mathrm{H}$ spectra were predicted to facilitate their future identification.

\section{ACKNOWLEDGMENT}

This work was supported by the Drug Institute, Warsaw. Dr. Michał H. Jamróz (Industrial Chemistry Research Institute, Warsaw) is gratefully acknowledged for writing a computer program for enumeration and code generation of the belt and Moebius isomers. The author also thank the reviewers for several important suggestions that lead to making this paper more complete and clear.

\section{APPENDIX}

To order the hexacene catacondensed isomers, let us introduce a code analogous to those introduced before. The first and the last six-membered rings are those involving the $(\mathrm{CH})_{4}$ moieties. Then, the first and the last rings do not differentiate catacondensed hexacene isomers and the four digit code, corresponding to the second, third, fourth, and fifth six-membered ring, can identify uniquely the catacondensed hexacene isomers, provided the equivalence between four-tuples includes the fact that the rings can be labeled in the opposite direction and that each four-tuple $\left(a_{2}, a_{3}, a_{4}, a_{5}\right)$ and $\left(\overline{\mathrm{a}}_{2}, \overline{\mathrm{a}}_{3}, \overline{\mathrm{a}}_{4}, \overline{\mathrm{a}}_{5}\right)$ is equivalent (where $\mathrm{a}_{\mathrm{i}}$ and $\overline{\mathrm{a}}_{\mathrm{i}}$ have the same meaning as before).

Now, we can introduce the ordering relation between the four-tuples: 
1. Selection of a representive code for a structure.

1.1. Among the equivalent codes representing a given structure we choose the code with the minimum sum of entries $\sum \mathrm{a}_{\mathrm{i}}$.

1.2. If there is more than one such code, we write them so that digit 1 is located at the entry with minimum position and digit 2 is not placed before it.

1.3. If there is no digit 1 in the code, we write the code so that the sequence of digits 0 at first entries is the longest.

1.4. Then we chose that code which, when read from left to right, forms the minimum decimal number.

\section{Ordering the codes}

2.1. The code with the greater number of digits 1 precedes the code with the smaller number of digits 1 .

2.2. When the number of digits 1 is the same the code with the longer series of neighboring digits 1 precedes the other.

2.3. When the number of digits 1 and the series of neighboring digits 1 are the same, then the code which forms the greater decimal number precedes the other.

Examples:

Ad. 1.1. From among codes representing the same structure (1200) and (1022) the former is chosen because the sum of the entries is smaller.

Ad. 1.2. The codes (0120) and (0210) represent the same structure; however, the former has digit 1 at the entry with the lower label.

Ad. 1.3. From among two codes (0020) and (0200) we chose the first one, for which the sequence of digit 0 at the entries with small labels is greater.

Ad. 1.4. The codes (1021) and (1201) represent the same structure; however, the first one forms a number 1021 which is smaller than 1201 .

Ad. 2.1. The code (1101) is smaller than (1020) because, in the first one, the number of digits 1 is greater.

Ad. 2.2. The code (1110) is smaller than the code (1101) because the former has three, whereas the latter has two neighboring digits 1 in a series.

Ad. 2.3. The code (1012) is greater than (1021) because the number 1012 is smaller than the number 1021 .

\section{REFERENCES AND NOTES}

(1) Franck, H. G.; Stadelhofer, J. W. Industrielle Aromatenchemie. Rohstoffe, Verfahren, Produkte; Springer: Berlin, Heidelberg, New York, 1987.

(2) Murdoch, J.; Geissman, T. A. Am. Miner. 1967, 52, 611. Blumer, M. Chem. Geol. 1975, 16, 247.

(3) Kroto, H. W.; Heath, J. R.; O'Brien, S. C.; Curl, R. F.; Smalley, R. E. Nature (London) 1985, 318, 162 .
(4) Schmalz, T. G.; Seitz, W. A.; Klein, D. J.; Hite, G. E. J. Am. Chem Soc. 1988, 110, 1113

(5) Kirby, E. C.; Pisanski, T. J. Math. Chem. 1998, 23, 151

(6) Subramoney, S. Adv. Mater. 1998, 10, 1157-1171.

(7) Kohnke, F. H.; Slavin, A. M.; Stoddart, J. F.; Williams, D. J. Angew. Chem. 1987, 99, 941-943. Angew. Chem., Int. Ed. Engl. 1987, 26, 892-894.

(8) Ashton, P. R.; Isaacs, N. S.; Kohnke, F. H.; Slavin, A. M.; Spencer, C. M.; Stoddart, J. F.; Williams, D. J. Angew. Chem. 1988, 100, 981983. Angew. Chem., Int. Ed. Engl. 1988, 27, 966-968.

(9) Stoddart, J. F. J. Inclusion Phenom. Mol. Recognit. Chem. 1989, 7, $2427-2435$.

(10) Mathias, J. P.; Stoddart, J. F. Chem. Soc. Rev. 1992, 21, 215-225.

(11) Kivelson, S.; Chapman, O. L. Phys. Rev. B 1983, 28, 7236

(12) Haase, M. A.; Zoellner, R. W. J. Org. Chem. 1992, 57, 1031-1033.

(13) Vögtle, F. Top. Curr. Chem. 1983, 115, 157-159.

(14) Alder, R. W.; Sessions, R. B. J. Chem. Soc., Perkin Trans II 1985 $1849-1854$

(15) Alder, R. W.; Allen, P. R., Edwards, L. S.; Fray, G. I.; Fuller, K. E.; Gore, P. M. Hext, N. M. Perry, M. H., Thomas, A. R.; Turner, K. S J. Chem. Soc., Perkin Trans I 1994, 3071-3077.

(16) Schröder, A.; Meckelburger, H.-B.; Vögtle, F. Top. Curr. Chem. 1994, 172, 179-201.

(17) Spartan version 5.0; Wavefunction, Inc.: 18401 Von Karman Ave., \#370, Irvine CA 92715 U.S.A. 8 Wavefunction, Inc.

(18) Gaussian 98 (Revision A.4); Frisch, M. J.; Trucks, G. W.; Schlegel, H. B.; Scuseria, G. E.; Robb, M. A.; Cheeseman, J. R.; Zakrzewski, V. G.; Montgomery, J. A.; Stratmann, R. E.; Burant, J. C.; Dapprich, S.; Milliam, J. M.; Daniels, A. D.; Kudin, K. N.; Strain, M. C.; Farkas, O.; Tomasi, J.; Barone, V.; Cossi, M.; Cammi, R.; Mennucci, B.; Pomelli, C.; Adamo, C.; Clifford, S.; Ochterski, J.; Petersson, G. A.; Ayala, P. Y.; Cui, Q.; Morokuma, K.; Malick, D. K.; Rabuck, A. D.; Raghavachari, K.; Foresman, J. B.; Cioslowski, J.; Ortiz, J. V.; Stefanov, B. B.; Liu, G.; Liashenko, A.; Piskorz, P.; Komaromi, I.; Gomperts, R.; Martin, R. L.; Fox, D. J.; Keith, T. A.; Al-Laham, M. A.; Peng, C. Y.; Nanayakkara, A.; Gonzales, C.; Challacombe, M.; Gill, P. M. W.; Johnson, B. G.; Chen, W.; Wong, M. W.; Andres, J. L.; Replogle, E. S.; Head-Gordon, M.; Reploge, E. S.; Pople, J. A. Gaussian, Inc.: Pittsburgh, PA, 1998.

(19) Hansen, A. E.; Bouman, T. D. J. Chem. Phys. 1985, 82, 5035.

(20) Woli?ski, K. J.; Hinton, F.; Pulay, P. J. Am. Chem. Soc. 1990, 112, 8251.

(21) Barszczewicz, A.; Jaszuński, M.; Jackowski, K. Chem. Phys. Lett. 1993, 203, 404

(22) Jackowski, K.; Barszczewicz, A.; Woźniak, K. Solid State NMR 1993 , $2,265$.

(23) Reference 23 deleted in press.

(24) Pecul, M.; Jackowski, K.; Woïniak, K.; Sadlej, J. Solid State NMR 1997, 8 .

(25) Mazurek, A. P.; Kozerski, L.; Sadlej, J.; Kawẹcki, R.; Bednarek, E.; Sitkowski, J.; Dobrowolski, J. Cz.; Maurin, J. K.; Biniecki, K.; Witowska, J.; Fiedor, P.; Pachecka, J. J. Chem. Soc., Perkin 21998 1223-1230.

(26) Bednarek, E.; Dobrowolski, J. Cz.; Dobrosz-Teperek, K.; Sitkowski, J.; Kozerski, L.; Lewandowski, W.; Mazurek, A. P. J. Mol. Struct. 2000, $554(2-3), 233-243$.

(27) Spectral Atlas of Polycyclic Aromatic Compounds; Kracher, W., Elison, S., Ewald, M., Garrigues, P., Gevers, E., Jacob, J., Eds.; Kluver Academic Publishers: Dordrecht, Boston, London, 1988; Vol. 2. 